\title{
Evaluación de la Proliferación de Células Madres Mesenquimales Estimuladas con Diferentes Intensidades de Ultrasonido de Baja Intensidad
}

\author{
Evaluation of Mesenchymal Stem Cell Proliferation \\ Using Different Low Intensity Pulsed Ultrasound Intensities
}

"Daniela Becerra G.; ** Jaime Díaz Z.; ${ }^{* * *}$ Flavio Carrión A.; ${ }^{* * * * *}$ Carolina Inostroza S. \& ***** Rodrigo Oyonarte W.

BECERRA, G. A.; DÍAZ, Z. J.; CARRIÓN, A. F.; INOSTROZA, S. C. \& OYONARTE, W. R. Evaluación de la proliferación de células madres mesenquimales estimuladas con diferentes intensidades de ultrasonido de baja intensidad. Int. J. Morphol., 30(2):621$626,2012$.

RESUMEN: Las Células Madres Mesenquimales (CMM) pueden ser afectadas en su capacidad de proliferar in vitro bajo estimulación física o bioquímica, siendo esta una capacidad esencial para un adecuado cultivo celular. Un método de estimulación física que ha demostrado ser eficiente en este sentido es el Ultrasonido Pulsátil de Baja Intensidad (USBI), aplicado en intensidades iguales o inferiores a $100 \mathrm{~mW} / \mathrm{cm}^{2}$, habitualmente entre 30 y $50 \mathrm{~mW} / \mathrm{cm}^{2}$. El objetivo de esta investigación fue determinar el nivel de intensidad de ultrasonido pulsátil de baja intensidad óptimo entre 30 y $50 \mathrm{~mW} / \mathrm{cm}^{2}$ para estimular la proliferación de CMM de médula ósea de ratas Sprague Dawley, in vitro. CMM $\left(1 \times 10^{6} \mathrm{cls} / \mathrm{kg}\right)$ de medula ósea de rata Sprague-Dawley fueron cultivadas (alfa-MEM, 20\%FBS y $1 \%$ antibiotico) y estimuladas con USBI (0,02 milisegundos), por 20 minutos dos veces al día por 10 días con intensidades de 0,30 y $50 \mathrm{~mW} / \mathrm{cm}^{2}$ (grupos A [control], B, C respectivamente). Se contabilizó el número de células en el cultivo y se evaluó morfología celular en microscopio óptico. Se utilizaron tests de ANOVA on Ranks y Bonferroni. Los cultivos estimulados con USBI presentaron mayores recuentos celulares, y se observaron diferencias estadísticamente significativas entre los grupos A y C ( $<<0,05)$. Se observaron diferencias morfológicas entre células de grupos estimulados con USBI y el control. La estimulación de las CMM en cultivos bidimensionales con USBI influencia cambios en la morfología celular y se concluye que $50 \mathrm{~mW} / \mathrm{cm}^{2}$ es la intensidad óptima dentro de las evaluadas para producir aumento en la proliferación celular ( $<<0.05$ ).

PALABRAS CLAVE: Células madre mesenquimales; ultrasonido.

\section{INTRODUCCIÓN}

Las Células Madres son precursoras de las células que existen en el cuerpo humano, cumpliendo un rol fundamental en el desarrollo embrionario (Caplan, 1991; Meyer \& Wiesmann, 2006), y en el potencial regenerativo de los tejidos (Tokalov et al., 2007). Una vez diferenciados los tejidos su presencia se hace más escasa, no obstante lo cual numerosos estudios han reportado su aislamiento en tejidos diferenciados, tanto en médula ósea (Friedenstein et al., 1987; Javason et al., 2001; Rhodes et al., 2004; Tropel et al., 2004), como en otros tejidos (Nathan et al., 2003; de Ugarte et al., 2003; Nöth et al., 2002; Fickert et al., 2003; Baksh et al., 2004; Jo et al., 2007; Dualibi et al., 2008; Yao et al., 2008) habiendo sido también descritas en cartílago, hueso subcondral y en tejido conectivo perivascular del cóndilo articular mandibular (Rabie et al., 2002; 2003).
La posibilidad de obtener células madres desde diferentes tejidos ha incentivado el desarrollo de técnicas para aislamiento y cultivo celular, para su posterior aplicación en terapias regenerativas. Para lo anterior, las capacidades de diferenciación, migración y crecimiento de un determinado grupo celular resultan claves en su potencial uso en terapias regenerativas. Estas pueden optimizarse con estimulación bioquímica y/o física. En efecto, el ambiente biomecánico resulta ser un potente regulador del comportamiento de las CMM de médula ósea en cultivos celulares (Malone et al., 2005). Dentro de los estímulos físicos se han estudiado entre otros el ultrasonido pulsátil de baja intensidad (USBI) (De Oliveira et al., 2008; Walker et al., 2007) y ultrasonido continuo (Choi et al., 2007; Lee et al., 2007; Cui et al., 2006, 2007).

\footnotetext{
Cirujano Dentista. Docente de Morfología, Unidad de Histología y Anatomía Topográfica, Facultad de Odontología, Universidad de los Andes, Santiago, Chile. Cirujano Dentista. Alumno de Magister en Ciencias Odontológicas mención Periodontología, Santiago, Chile.

**** Bioquímico, Doctor en Ciencias Biológicas, Director de Laboratorio de Inmunología y Terapia Celular, Facultad de Medicina, Universidad de Los Andes, Santiago, Chile.

***** Bioquímica, Magíster en Microbiología. Centro de Investigación de Biología y Regeneración Oral (CIBRO), Fac. de Odontología, Universidad de los Andes, Santiago, Chile.

****** Cirujano Dentista, Máster en Ciencias, Ortodoncista. Profesor de Ortodoncia, Facultad de Odontología, Universidad de Los Andes, Santiago de Chile, Santiago, Chile. Financiamiento: Proyecto FIC-ODO 2008, Facultad de Odontología, Universidad de los Andes.
} 
El USBI es una forma de energía mecánica transmitida a través de los tejidos a niveles de energía inferiores a $0.1 \mathrm{~W} / \mathrm{cm} 2$ (Schortinghuis et al., 2003). Su uso ha sido extensamente estudiado en medicina para la aceleración de la velocidad de reparación de fracturas óseas (Kristiansen et al., 1997; Busse et al., 2009). A su vez, se han demostrado efectos positivos del USBI en el crecimiento endocondral tanto in vivo como in vitro (Choi et al.; El-Bialy et al., 2003) incluyendo variaciones en el patrón de crecimiento endocondral del proceso condilar mandibular en animales de experimentación (El-Bialy et al., 2003; El-Bialy, 2007; Oyonarte et al., 2009). En cultivos celulares, el USBI ha demostrado ser de utilidad estimulando la diferenciación de condrocitos (El-Bialy et al., 2010; Saito et al., 2004) y osteoblastos (Kristensen et al.), la formación de matriz extracelular ósea (Tsai et al., 1992; Suzuki et al., 2009), aumentando la expresión de proteína morfogenética ósea (Suzuki et al., 2009) y la proliferación celular (El-Bialy et al., 2010), e inhibiendo procesos de apoptosis en cultivos de CMM (Lee et al.). Los parámetros físicos dentro de los que el USBI es aplicado en condiciones de cultivo celular de CMM pueden variar ampliamente dentro del rango de emisión de energía definido como ultrasonido pulsátil de baja intensidad, bajo $0,1 \mathrm{~W} / \mathrm{cm}^{2}$, habitualmente fluctuando entre $30 \mathrm{~mW} / \mathrm{cm}^{2}$ y $50 \mathrm{~mW} / \mathrm{cm}^{2}$. Resulta de interés determinar el efecto de la aplicación de USBI en intensidades de $30 \mathrm{~mW} / \mathrm{cm}^{2}$ y $50 \mathrm{~mW} / \mathrm{cm}^{2}$ sobre la proliferación celular de cultivos de CMM.

El objetivo de este estudio fue determinar el efecto de dos niveles de intensidad de ultrasonido pulsátil de baja intensidad sobre la proliferación de CMM de médula ósea de ratas Sprague Dawley, in vitro.

\section{MATERIAL Y MÉTODO}

Purificación y cultivo de CMM de médula ósea de ratas. CMM de médula ósea de fémur de ratas Sprague - Dawley macho de 200 a 250 gramos de peso y 7 semanas de edad fueron aisladas según el siguiente protocolo: la médula ósea se aspiró con una jeringa de $10 \mathrm{~mL}$ de volumen y se resuspendió en un medio completo compuesto por a-MEM (Minimal Eagle Medium, GIBCO, Invitrogen), 20\% de suero bovino fetal (FBS [MSC Qualified Fetal Bovine Serum, 100 $\mathrm{mL}$, GIBCO, Invitrogen]) y $1 \%$ de Antibiótico (PenicilinaEstreptomicina, PenStrep, GIBCO, Invitrogen).

Las células obtenidas de la médula ósea se centrifugaron en un tubo Falcon ${ }^{\circledR}$ (Blue maxim, $50 \mathrm{~mL}$, Polypropylene Conical Tube, Falcon, Becton Dickinson) de $50 \mathrm{~mL}$ con Ficoll@ (Ficoll-PaqueTM PLUS, GE Healthcare,
Fermelo Biotec) durante 15 minutos, a $2000 \mathrm{rpm}$ y $23^{\circ} \mathrm{C}$, obteniéndose una banda de células mononucleares, dentro de las cuales se encuentran las CMM. Posteriormente las CMM se purificaron resuspendiéndose en un tubo de $15 \mathrm{~mL}$ con PBS 1x (Dulbecoo's Phosphate - Buffered Saline powder, GIBCO, Invitrogen) y centrifugando durante 10 minutos a $1680 \mathrm{rpm}$ y $23^{\circ} \mathrm{C}$.

Obtenidas las CMM, se resuspendieron en $3 \mathrm{~mL}$ de medio completo, se contaron en una cámara de Neubauer (Neubauer Improved Pright Line, 0,1 $\mathrm{mm}$ de profundidad, $0,0025 \mathrm{~mm} 2, \mathrm{HBG}$ ) y se sembraron en placas de 6 pocillos a una concentración de 1 x $106 \mathrm{cls} / \mathrm{mL}$.

Se cultivaron durante 3 semanas hasta alcanzar la confluencia cercana al $80 \%$ y se tripsinizaron con $1 \mathrm{~mL}$ de Tripsina (TrypLETM Select, GIBCO, Invitrogen) durante 5 minutos, para posteriormente, diluir en PBS $1 \mathrm{x}$ y centrifugar a $1680 \mathrm{rpm}$ por 5 minutos a $23^{\circ} \mathrm{C}$. De este modo las CMM entran al pasaje 1 y se cultivan hasta el pasaje 4 . Las células obtenidas tras los pasajes se contaron y sembraron en 3 placas de 6 pocillos a una concentración de 1x $105 \mathrm{cls} / \mathrm{mL}$.

Caracterización de CMM de médula ósea de ratas. Para la caracterización in vitro se utilizaron los criterios publicados según la International Society for Cellular Therapy (ISCT): adherencia al plástico (Friedstein et al., 1987), proliferación, morfología fibroblastoide (Fibroblast like-cells) y diferenciación a linaje condrogénico, osteogénico y adipogénico (Tokalov et al.). Se realizaron las diferenciaciones a cada linaje según protocolos descritos en el Technical Manual, Stemcell Technologies (2008), versión 1.2.0.

Estimulación de CMM con ultrasonido de baja intensidad. Se utilizó un aparato de Ultrasonido Medlinne 4100 Terapia Combinada (Santiago, Chile), calibrado para emisión de USBI específicamente para este estudio. Se dividieron las placas en tres grupos de estudio, donde se utilizaron intensidades de 0,30 y $50 \mathrm{~mW} / \mathrm{cm}^{2}$, con pulsos cada 0,2 milisegundos, aplicados durante 20 minutos, dos veces por día y hasta la confluencia de las CMM al $80 \%$. En cada placa se sembraron 4 pocillos con CMM en pasaje 4 a una concentración de $1 \times 10^{6} \mathrm{cls} / \mathrm{mL}$.

Evaluación de cambios cualitativos y cuantitativos de CMM. Se capturaron imágenes digitales utilizando un microscopio óptico con aumento de 40X, obteniéndose imágenes digitales para cada cultivo. Las imágenes de las células fueron analizadas utilizando el programa SigmaScan Pro 5.0, donde se registraron los cambios morfológicos de cada grupo de células y el número de estas por superficie. Se realizó el recuento celular el día 7 y se evaluó el porcentaje de confluencia. 
BECERRA, G. A.; DÍAZ, Z. J.; CARRIÓN, A. F.; INOSTROZA, S. C. \& OYONARTE, W. R. Evaluación de la proliferación de células madres mesenquimales estimuladas con diferentes intensidades de ultrasonido de baja intensidad. Int. J. Morphol., 30(2):621-626, 2012.

Análisis estadístico. Se aplicaron para el análisis de los datos los test estadísticos de ANOVA on ranks y Bonferroni.

\section{RESULTADOS}

La aislación, cultivo y caracterización de CMM de médula ósea de fémur de rata Sprague - Dawley se efectuó según el protocolo descrito. Se obtuvo una cantidad aproximada de 3,5 $\mathrm{x}$ $10^{7} \mathrm{cls} / \mathrm{cm}^{2}$ iniciales, cantidad suficiente para lograr cultivos de $3,0 \times 10^{6} \mathrm{cls} / \mathrm{cm}^{2}$ y realizar los experimentos in vitro.

El estudio concluyó el día 7 donde el primer grupo en confluir al 80\% fue el de las CMM estimuladas con USBI al 50 $\mathrm{mW} / \mathrm{cm}^{2}$.
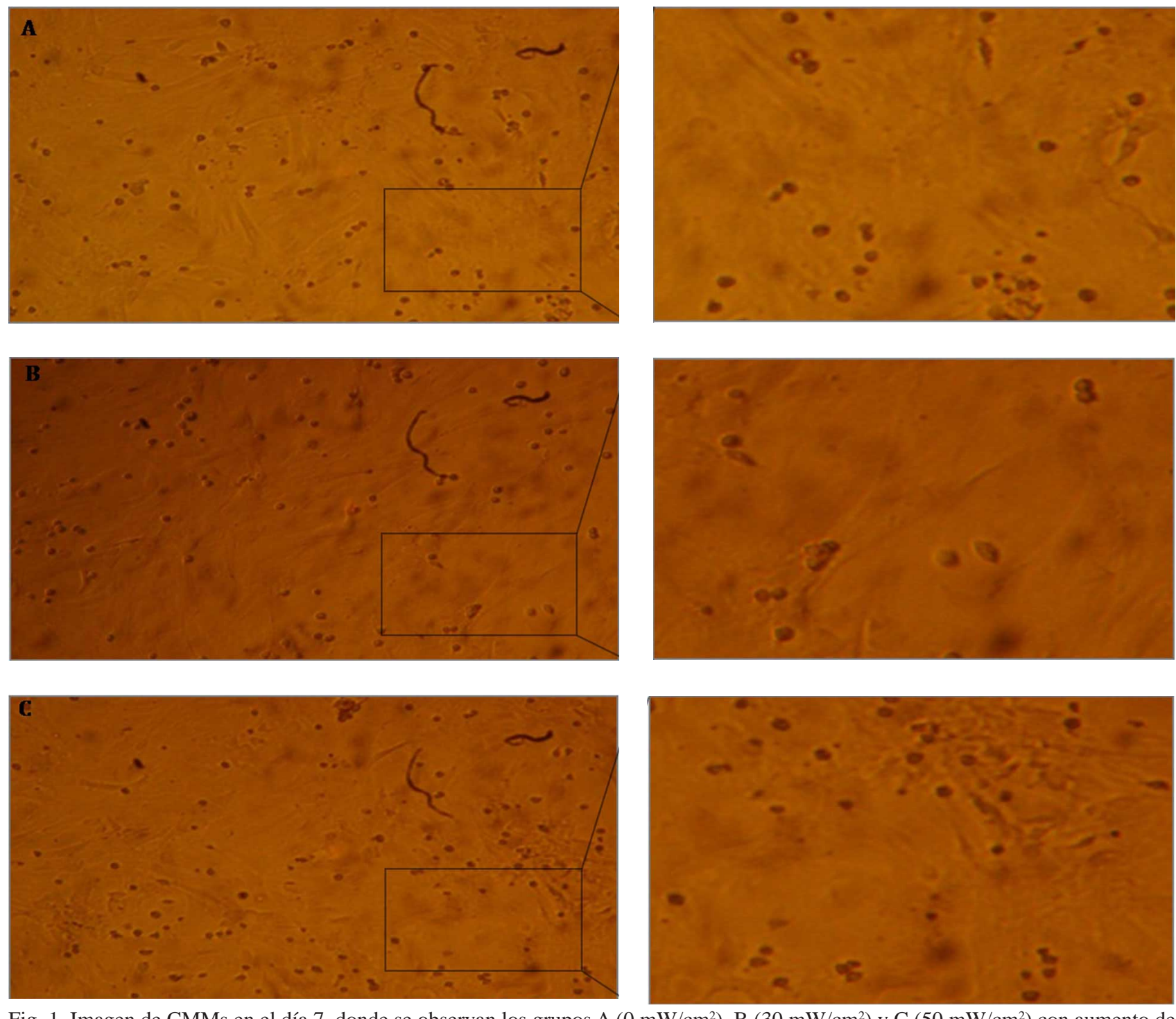

Fig. 1. Imagen de CMMs en el día 7, donde se observan los grupos A $\left(0 \mathrm{~mW} / \mathrm{cm}^{2}\right), \mathrm{B}\left(30 \mathrm{~mW} / \mathrm{cm}^{2}\right)$ y C $\left(50 \mathrm{~mW} / \mathrm{cm}^{2}\right)$ con aumento de $200 X$.

Se observaron diferencias morfológicas entre el control y los grupos estimulados con USBI. En el control, las células presentaron una morfología alargada y aplanada, semejante a fibroblastos. Los grupos estimulados presentaron una morfología más amorfa (Fig. 1).

A su vez, los cultivos estimulados con 30 y 50 $\mathrm{mW} / \mathrm{cm}^{2}$ presentaron un mayor número de células por unidad de superficie en comparación al grupo control (19, 31 y 33 x 103 células por $\mathrm{cm}^{2}$ respectivamente), constatándose diferencias estadísticamente significativas (ANOVA on Ranks, $p<0,05$ ). Esta diferencia en proliferación celular fue significativamente mayor en placas estimuladas con $50 \mathrm{~mW} / \mathrm{cm}^{2}$ de intensidad, respecto al grupo control (Bonferroni, $\mathrm{p}=0,037$ ), como se observa en la Fig 2.
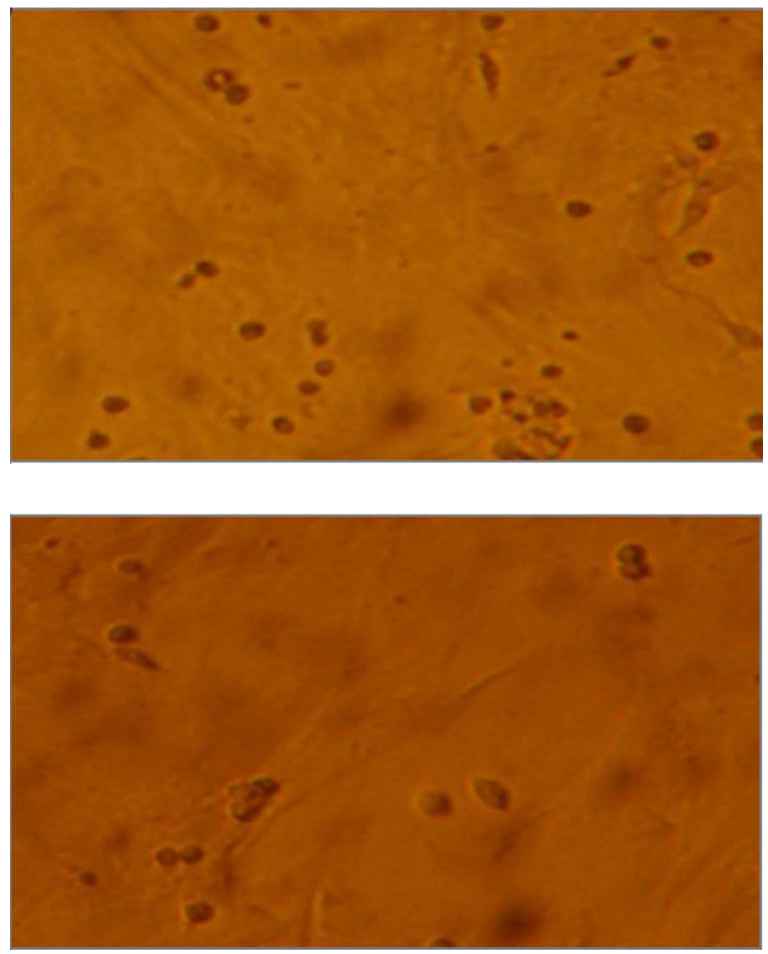


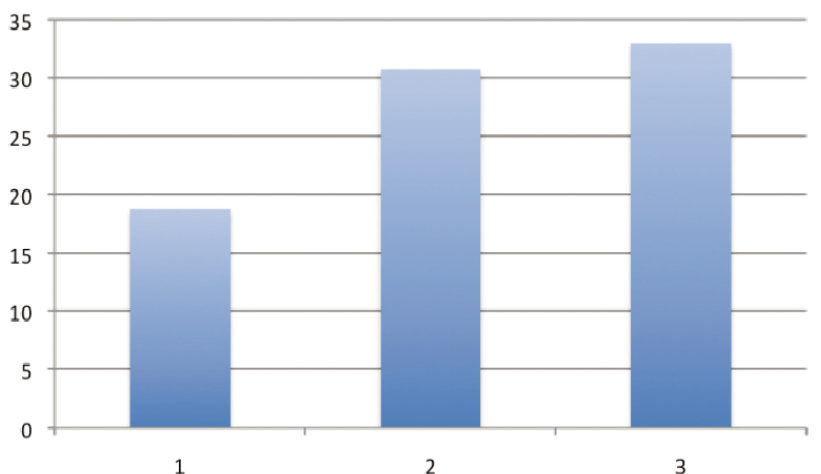

Fig. 2. Proliferación celular promedio expresada $\times 10^{3}$ células por $\mathrm{cm}^{2}$. Grupos 1: control $0 \mathrm{~mW} / \mathrm{cm}^{2}, 2: 30,3: 50 \mathrm{~mW} / \mathrm{cm}^{2}$, observándose diferencias significativas entre grupos 1 y 3 .

\section{DISCUSIÓN}

Este estudio tuvo por objetivo establecer la intensidad óptima entre dos intensidades de estimulación con USBI en cultivos bidimensionales de CMM de médula ósea de ratas Sprague-Dawley, identificando sus efectos sobre la proliferación y morfología celular. Así, se determinó que la intensidad a la que se observaron diferencias estadísticamente significativas fue la de $50 \mathrm{~mW} / \mathrm{cm}^{2}$ en relación a los controles. Ello ofrece un parámetro que permite favorecer la proliferación de CMM de rata de médula ósea. Estos resultados sugieren que la utilización de USBI (30 $\mathrm{mW} / \mathrm{cm}^{2}$ y $50 \mathrm{~mW} / \mathrm{cm}^{2}$ ) durante el proceso de cultivo favorece los procesos de proliferación in vitro, siendo preferible la intensidad de $50 \mathrm{~mW} / \mathrm{cm}^{2}$.

Nuestros resultados concuerdan con los de otros autores que han estudiado los efectos in vitro de la estimulación con USBI en cultivos celulares sobre variables como crecimiento, diferenciación o migración celular en que la aplicación de USBI favorece dichos procesos (De Oliveira et al.; Walker et al.). De hecho, se ha reportado que el USBI promueve la diferenciación de CMM a condrocitos (Lee et al.; Cui et al., 2006, 2007). La explicación a dicho fenómeno está aún en un plano teórico, e involucraría el rol mecanotransductivo de integrinas transmembrana frente a la estimulación del cultivo celular con USBI (Lee et al.). Estas estimularían la proliferación, diferenciación y migración celular, y además, generarían un proceso antiapoptótico que aumentaría la viabilidad celular (Lee et al.; Mobasheri et al., 2002).

Distintos autores han empleado diferentes intensidades de emisión de USBI, preferentemente a $30 \mathrm{~mW} / \mathrm{cm}^{2}$ para estudios in vitro (Appleford et al., 2007; Suzuki et al.) u otras intensidades dentro de lo que es caracterizado como USBI (Tien et al., 2008; Zhang et al., 2003). Resulta aparente que diferencias asociadas al tipo de aparato de US utilizado, características de área y estructura del transductor, así como la frecuencia de emisión, entre otros, podrían influir en el establecimiento de niveles óptimos de intensidad de emisión de USBI.

La aplicación de USBI en cultivos celulares representa una modalidad sencilla de utilización métodos de estimulación biofísica durante el cultivo de CMM, facilitando su proliferación y optimizando la eficiencia de dicho proceso, habiendo ya sido exitoso su uso en CMM de origen orofacial (Appleford et al.). Los presentes resultados sugieren que la utilización de USBI $\left(30 \mathrm{~mW} / \mathrm{cm}^{2}\right.$ y $50 \mathrm{~mW} /$ $\mathrm{cm}^{2}$ ) durante el proceso de cultivo favorece los procesos de proliferación in vitro, siendo este fenómeno de mayor relevancia a intensidades de $50 \mathrm{~mW} / \mathrm{cm}^{2}$.

En conclusión, la aplicación de USBI en cultivos bidimensionales de CMM de médula ósea favorece la proliferación celular, variando a su vez la morfología de las células estimuladas con USBI.

La estimulación de cultivos celulares de CMM de médula ósea de rata Sprague-Dawley a una intensidad de $50 \mathrm{~mW} / \mathrm{cm}^{2}$ es más eficiente que la intensidad de $30 \mathrm{~mW} /$ $\mathrm{cm}^{2}$ como factor coadyuvante de la proliferación celular, bajo las condiciones aplicadas en este estudio.

\section{AGRADECIMIENTOS}

Agradecemos a Facultad de Odontología de la Universidad de los Andes por el apoyo y financiamiento y a Fabiola Díaz por ayudarnos en la manipulación de cultivos celulares y por el manejo y cuidado de las células madres mesenquimales.

BECERRA，G. A.; DÍAZ，Z. J.; CARRIÓN，A. F.; INOSTROZA, S. C. \& OYONARTE, W. R. Evaluation of mesenchymal stem cell proliferation using different low intensity pulsed ultrasound intensities. Int. J. Morphol., 30(2):621-626, 2012 .

SUMMARY: Mesenchymal Stem Cells (MSCs) can be affected in their capabilities to proliferate in vitro under physical and/or biochemical stimulation. The aim of this study was to select an optimal intensity level for low intensity pulsed ultrasound (LIPUS) stimulation of Sprague-Dawley bone marrow MSCs proliferation in vitro. Bone marrow MSCs of Sprague-Dawley rats 
where cultured (a-MEM, 20\%FBS and 1\%antibiotic) and stimulated with LIPUS (0,02 milisec), for 20 minutes twice daily for 10 days, at intensities of 0 (control), 30 and $50 \mathrm{~mW} / \mathrm{cm} 2$ (groups A, B, C). Cellular count and morphological evaluation were performed. ANOVA and Bonferroni tests were performed. LIPUS-stimulated cultures displayed greater cellular counts, and significant differences were observed between groups $\mathrm{A}$ and $\mathrm{C}(\mathrm{p}<0,05)$. Morphological differences were observed between cells from LIPUS-stimulated and control groups. An intensity of $50 \mathrm{~mW} / \mathrm{cm} 2$ elicits increased cellular proliferation $(\mathrm{p}<0.05)$. Stimulation of MSCs cultures with LIPUS influences cellular morphology.

\section{cells; ultrasound.}

KEY WORDS: Adult stem cells; Mesenchymal stem

\section{REFERENCIAS BIBLIOGRÁFICAS}

Appleford, M. R.; Oh, S.; Cole, J. A.; Protivinsky, J. \& Ong, L. Ultrasound effect on osteoblast precursor cells in trabecular calcium phosphate scaffolds. Biomaterials, 28(32):4788-94, 2007.

Baksh, D.; Song, L. \& Tuan, R. S. Adult mesenchymal stem cells: characterization, differentiation and application in cell and gene therapy. J. Cell. Mol. Med., 8(3):301-16, 2004.

Busse, J. W.; Kaur, J.; Mollon, B.; Bhandari, M.;Tornetta, P. 3rd.; Schünemann, H. J. \& Guyatt, G. H. Low intensity pulsed ultrasonography for fractures: systematic review of randomised controlled trials. BMJ, 27:338:b351, 2009.

Caplan, A. I. Mesenchymal stem cells. J. Orthop. Res., 9(5):64160, 1991.

Choi, B. H.; Choi, M. H.; Kwak, M. G.; Min, B. H.; Woo, Z. H. \& Park, S. R. Mechanotransduction pathways of low - intensity ultrasound in C - 28/I2 human chondrocite line. Proc. Inst. Mech. Eng. H., 221(5):527-35, 2007.

Cui, J. H.; Park, K.; Park, S. R. \& Min, B. H. Effects of Low Intensity Ultrasound on Chondrogenic Differentiation of Mesenchymal Stem Cells Embedded in Polyglycolic Acid: An in vivo Study. Tissue Eng., 12(1):75-82, 2006.

Cui, J. H.; Park, S. R.; Park, K.; Choi, B. H. \& Min, B. H. Preconditionind of mesenchymal stem cells with low intensity ultrasound for cartilage formation in vivo. Tissue Eng., 13(2):351-60, 2007.

De Oliveira, R. F.; Oliverira, D. A.; Monteiro, W.; Zangaro, R. A.; Magini, M. \& Soarez, C. P. Comparison between the effect of low - level ser therapy and low - intensity pulsed ultrasonic irradiation in vitro. Photomed. Laser Surg., 26(1):6-9, 2008.

De Ugarte, D. A.; Alfonso, Z.; Zuk, P. A.; Elbarbary, A.; Zhu, M.;
Ashjian, P.; Benhaim, P.; Hedrick, M. H. \& Fraser, J. K. Differential expression of stem cell mobilization-associated molecules on multi-lineage cells from adipose tissue and bone marrow. Inmunol. Lett., 89(2-3):267-70, 2003.

Dualibi, S. E.; Dualibi, M. T.; Zhang, W.; Asrican, R.; Vacanti, J. P. $\&$ Yelick, P. C. Bioengineered dental tissues grown in the rat jaw. J. Dent. Res., 87(8):745-50, 2008.

El-Bialy, T. Therapeutic Ultrasound applications in craniofacial growth, healing and tissue engineering. Rejuvenation Res., 10(3):367-71, 2007

El-Bialy, T.; El-Shamy, I. \& Graber, T. M. Growth modification of the rabbit mandible using therapeutic ultrasound: Is it possible to enhance functional appliance results? Angle Orthod., 73(6):631-9, 2003.

El-Bialy, T.; Uludag, H.; Jomha, N. \& Badylak, S. F. In vivo Ultrasound- Assisted Tissue- Engineered Mandibular Condyle: A pilot study in rabbits. Tissue Eng. Part C Metods, 16(6):131523,2010

Fickert, S.; Fiedler, J. \& Brenner, R. E. Identification, quantification and isolation of mesenchymal progenitor cells from osteoarthritic synovium by fluorescence automated cell sorting. Osteoarthritic Cartilage, 11(11):790-800, 2003.

Friedenstein, A. J.; Chailakhyan, R. K. \& Gerasimov, U. V. Bone Marrow osteogenic stem cells: in vitro cultivation and transplantation in diffusion chambers. Cell. Tissue Kinet., 20(3):263-72, 1987

Javason, E. H.; Colter, D. C.; Schwartz, E. J. \& Prockop, D. J. Rat marrow stromal cells are more sensitive to plating density and expand more rapidly from single - cell - derived colonies than human marrow stromal cells. Stem Cells, 19(3):219-25, 2001.

Jo, Y. Y.; Lee, H. J.; Kook, S. Y.; Choung, H. W.; Park, J. Y.; Chung, J. H.; Choung, Y. H.; Kim, E. S.; Yang, H. C. \& Choung, P. H. Isolation and characterization of postnatal stem cells from human dental tissues. Tissue Eng., 13(4):767-73, 2007.

Kristiansen, T. K.; Ryaby, J. P.; McCabe, J.; Frey, J. J. \& Roe, L. R. Accelerated healing of distal radial fractures with the use of specific, low-intensity ultrasound. A multicenter, prospective, randomized, double-blind, placebo-controlled study. J. Bone Joint Surg. Am., 79(7):961-73, 1997.

Lee, H. J.; Choi, B. H.; Min, B. H. \& Park, S. R. Low intensity ultrasound inhibits apoptosis and enhances viability of human mesenchymal stem cells in three - dimensional alginate culture during chondrogenic differentiation. Tissue Eng., 13(5):104957, 2007.

Malone, A. M.; Narain, R. \& Jacobs, C- R. Biomechanical regulation of mesenchymal stem and progenitor cells and the implications for regenerative medicine. Curr. Opin. Orthop., 16(5):363-7, 2005. 
Meyer, U. \& Wiesmann, H. P. Bone and Cartilage engineering. Berlin, Springer, 2006.

Mobasheri, A.; Carter, S. D.; Martín-Vasallo, P. \& Shakibael, M. Integrins and stretch activated ion channels; putative components of functional cell surface mechanoreceptiors in articular chondrocytes. Cell Biol. Int., 26(1):1-18, 2002.

Nathan, S.; Das De, S.; Thambyah, A.; Fen, C.; Goh, J. \& Lee, E. H. Cell based therapy in the repair of osteochondral defects: a novel use for adipose tissue. Tissue Eng., 9(4):733-44, 2003.

Nöth, U.; Osyczka, A. M.; Tuli, R.; Hickok, N. J.; Danielson, K. G. \& Tuan, R. S. Multilineage mesenchymal differentiation potential of human trabecular bone - derived cells. J. Orthop. Res., 20(5):1060-9, 2002.

Oyonarte, R.; Zarate, M. \& Rodriguez, F. Low Intensity Pulsed Ultrasound Stimulation of condylar growth in rats. Angle Orthod., 79(5):964-70, 2009.

Rabie, A. B.; Leung, F. Y.; Chayanupaktul, A. \& Hägg, U. The correlation between neovascularization and bone formation in the condyle during forward mandibular positioning. Angle Orthod., 72(5):431-8, 2002.

Rabie, M. A. B.; Chayanupaktul, A. \& Hägg, U. Stepwise advancement using fixed functional appliances: experimental perspective. Semin. Orthod., 9(1):41-6, 2003.

Rhodes, N. P.; Srivastava, J. K.; Smith, R. F. \& Longinotti, C. Heterogeneity in proliferative potential of ovine mesenchymal stem cells colonies. J. Mater. Sci. Mater. Med., 15(4):397-402, 2004.

Saito, M.; Soshi, S.; Tanaka, T. \& Fujii, K. Intensity-related differences in collagen post-translational modification in MC3T3-E1 osteoblasts after exposure to low- and highintensity pulsed ultrasound. Bone, 35(3):644-55, 2004.

Schortinghuis, J.; Stegenga, B.; Raghoebar, G. M. \& de Bont, L. G. Ultrasound stimulation of maxillofacial bone healing. Crit. Rev. Oral Biol. Med., 14(1):63-74, 2003.

Stemcell Technologies. Enumeration \& Expansion of mouse mesenchymal stem cells using mesencult $\AA$. Technical Manual, Version 1.2.0, STEMCELL Technologies, 2008.

Suzuki, A.; Takayama, T.; Suzuki, N.; Kojima, T.; Ota, N.; Asano, S. \& Ito, K. Daily low-intensity pulsed ultrasound stimulates production of bone morphogenetic protein in ROS 17/2.8 cells. J. Oral Sci., 51(1):29-36, 2009.

Tien ,Y. C.; Lin, S. D.; Chen, C. H.; Lu, C. C.; Su, S. J. \& Chih, T. T. Effects of pulsed low-intensity ultrasound on human child chondrocytes. Ultrasound Med. Biol., 34(7):1174-81, 2008.

Tokalov, S.; Grüner, S.; Schindler, S.; Iagunov, A.; Baumann, M.
\& Abolmaali, N. A number of bone marrow mesenchymal stem cells but neither phenotype nor differentiation capacities changes with age of rats. Mol. Cells, 24(2):255-60, 2007.

Tropel, P.; Noël, D.; Platet, N.; Legrand, P.; Benabid, A. L. \& Berger, F. Isolation and characterization of mesenchymal stem cells from adult bone marrow. Exp. Cell Res., 295(2):413-9, 2004.

Tsai, C. L.; Chang, W. H. \& Liu, T. K. Preliminary studies of duration and intensity of ultrasonic treatments on fracture repair. Chinese J. Physiol., 35(1):21-6, 1992.

Walker, N.; Denegar, C. \& Preische, J. Low-intensity pulsed ultrasound and pulsed electromagnetic field in the treatement of tibial fractures: a systematic review. J. Athl. Train., 42(4):530-5, 2007.

Yao, S.; Pan, F.; Prpic, V. \& Wise, G. E. Differentiation of Stem Cells in the dental follicle. J. Dental Res., 87(8):767-71, 2008.

Zhang, Z. J.; Huckle, J.; Francomano, C. A. \& Spencer, R. G. The effects of pulsed low-intensity ultrasound on chondrocyte viability, proliferation, gene expression and matrix production. Ultrasound Med. Biol., 29(11):1645-51, 2003.

Dirección de Correspondencia:

Rodrigo Oyonarte W.

Cirujano Dentista, Máster en Ciencias, Ortodoncista.

Profesor de Ortodoncia

Facultad de Odontología

Universidad de Los Andes

Santiago de Chile

CHILE

Email: royonarte@miuandes.cl

Recibido : 30-01-2012

Aceptado: 09-05-2012 